\title{
Genetic landscapes out of Africa
}

As the birthplace of modern humans, Africa holds unique significance for human population genetics. The continent is now home to approximately 900 million people in more than 2,000 different ethnolinguistic groups, and yet patterns of genetic variation in African populations have remained largely uncharacterized. A major study of genetic diversity in African and African American populations now helps to unravel the complex evolutionary history of these groups and human populations worldwide, and might lead to improvements in understanding population-specific disease risk.

Tishkoff and colleagues analysed variation in a panel of over 1,300 microsatellites and insertionsdeletions in 121 African, 4 African American and 60 non-African populations. This work has increased the scale of genetic analysis of African populations to include a much wider range of groups in a genome-wide study than has previously been done. This gave the authors sufficient data to explore patterns of genetic diversity both within and between populations, to construct phylogenetic trees to look at the genetic distances between populations (which could be compared with the geographic distances), and to explore the relationship between genetic and cultural distinctions in greater detail than before.

African and African American populations stand out in the worldwide comparisons as having the highest levels of within- and betweenpopulation diversity. This is consistent with divergent ancestral populations in Africa, compared with much smaller founder groups that were formed as humans migrated away from the continent. Indeed, diversity within populations generally decreases with increased distance from Africa. The authors found genetic evidence of 14 ancestral population clusters. Importantly, these analyses revealed more substructure within African populations than had previously been observed, which has implications for the appropriate design and interpretation of association studies for diseases and other traits.

The authors also used their genetic data to explore the population history of many ethnolinguistic groups, giving further insight into where migrations have occurred and which populations have remained relatively isolated. For example, considerable NigerKordofanian ancestry was found in nearly all populations, which probably reflects the spread and mixing with local populations of the farming Bantu-speaking group in the last $\sim 5,000$ years. Interestingly, although genetic clusters are generally consistent with language groups there are some exceptions such as the Maasai and Pygmies - for which cultural distinction has been robust despite genetic mixing.

A central theme that emerges from these analyses is the genetic complexity of African populations. The significance of this is twofold: it confirms that further genotyping and resequencing of African genomes is likely to be highly informative for dissecting the history of human evolution; and, importantly for current and future inhabitants of Africa, it emphasizes that ethnically diverse populations need to be included in studies of disease risk and drug response.

\section{Mary Muers}

ORIGINAL PAPER Tishkoff, S. A. et al. The genetic struture and history of Africans and African Americans. Science 30 Apr 2009 (doi:10.1126/science.1172257) FURTHER READING Pagel, M. Human language as a culturally transmitted replicator. Nature Rev. Genet. 12 May 2009 (doi:10.1038/nrg2560) 